\title{
Pediatric mesenteric panniculitis: three cases and a review of the literature
}

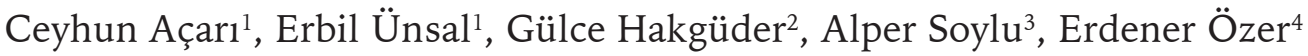 \\ Divisions of ${ }^{1}$ Pediatric Rheumatology and ${ }^{3}$ Nephrology, Departments of ${ }^{2}$ Pediatric Surgery and ${ }^{4}$ Pathology, Dokuz Eylül \\ University Faculty of Medicine, Izmir, Turkey.E-mail: ceyhun.acari@deu.edu.tr, ceyhun_acari@hotmail.com \\ Received: 17th September 2018, Revised: 14th November 2018, 25th December 2018, 17th January 2019, \\ Accepted: 17th January 2019
}

SUMMARY: Açarı C, Ünsal E, Hakgüder G, Soylu A, Özer E. Pediatric mesenteric panniculitis: three cases and a review of the literature. Turk J Pediatr 2019; 61: 798-803.

\begin{abstract}
Mesenteric panniculitis is an inflammatory and fibrotic process in the mesenteric adipose tissue with unknown etiology. It is rarely seen in general, particularly in children. Etiology is unknown, and pathophysiology is not clear. Factors that trigger the disease are malignancy, tuberculosis, trauma, medications and past surgical interventions. There is no pediatric case series in the literature except single case reports. This paper consists of 3 cases: The first case is a 5-month-old girl, the youngest patient in the literature, who was referred to a pediatric surgeon with vomiting and abdominal distention. She had diffused intraabdominal fluid and mesenteric panniculitis documented by perioperative biopsy. The second case had acute abdominal pain with perforated appendicitis, who eventually had mesenteric panniculitis in the evaluation of the pathological specimen. The last case had a diagnosis of polyarticular juvenile idiopathic arthritis (JIA), successfully treated with etanercept, and has been in remission for 2 years. Interestingly, in one of her routine visits, she had pallor, anemia and renal failure. Bilateral hydronephrosis was detected. Magnetic resonance imaging (MRI) of the abdomen revealed retroperitoneal fibrosis, and mesenteric panniculitis was the histopathological diagnosis.
\end{abstract}

Key words: mesenteric panniculitis, children, biologics.

Mesenteric panniculitis is an inflammatory and fibrotic process in the mesenteric adipose tissue with unknown etiology, which may be acute or chronic, and is rarely seen in children. Although sclerosing mesenteritis, retroperitoneal fibrosis and mesenteric lipodystrophy are used in literature, the most common definition is mesenteric panniculitis. ${ }^{1}$ It is commonly seen in the mesenteric fat tissue of small bowel and less frequently in the mesenteric fat tissue of large bowel. ${ }^{2}$ Several pathophysiological mechanisms such as past surgical history, trauma, hypoxia, allergy, infections and autoimmunity have been considered in the etiopathogenesis of mesenteric panniculitis. ${ }^{3}$
One of the mechanisms is monoclonal / oligoclonal B cell proliferation. Inflammation in the mesenteric panniculitis is linked to adipocytokines such as adiponectin, resistin, interleukin-6 (IL-6) and tumor necrosis factor-alpha (TNF-alpha). Macrophages found in the adipose tissue may be transformed from local preadipocytes. This supports the hypothesis that adipocytes and macrophages can be transformed into one another. ${ }^{4}$ There is only one case association with pediatric lupus in the English literature up to date. ${ }^{5}$ Histopathological diagnosis is accepted as the gold standard. ${ }^{6}$ It usually reveals chronic inflammation accompanied by fat necrosis, fibrosis, and lymphocyte infiltration. ${ }^{7}$ Recently,

This study was presented as the 4th National Pediatric Rheumatology Congress 4-7 April 2018 Bodrum and the 25th European Paediatric Rheumatology Congress 5-8th September 2018, Lisbon, Portugal. 
radiology is the most accessible, non-invasive diagnostic modality that is used in many new investigations. ${ }^{8}$ There is no consensus on the management and treatment of the disease, particularly in children. ${ }^{2,9}$

\section{Case 1}

A five-month-old girl was admitted to the hospital with irritability and vomiting that started in the morning. On physical examination; she had dehydration and abdominal distention, without bowel movement on auscultation. Laboratory tests revealed leukocytosis $\left(15500 / \mathrm{mm}^{3}\right)$, with mild elevation of erythrocyte sedimentation rate (ESR): $28 \mathrm{~mm} / \mathrm{h}$. Prothrombin time, active partial thromboplastin time, d-dimer were normal. Renal function tests, amylase, lipase, lipid levels, urine and blood amino acid levels, fatty acids, urinary organic acids, tandem-ms were found to be normal excluding a metabolic disease.

Immunoglobulin levels were found to be normal. Complement proteins (C3, C4), antinuclear antibody (ANA), anti-neutrophilic cytoplasmic antibody (ANCA) were negative. Direct abdominal X-ray was normal, but free fluid was detected on the abdominal ultrasonography. Abdominal computed tomography (CT) showed widespread free fluid. (Fig. 1) Radiologic findings were not specific for any diagnosis. Intestinal obstruction was suspected. Explorative laparotomy was applied for the differential diagnosis. Dense lipid deposits were observed

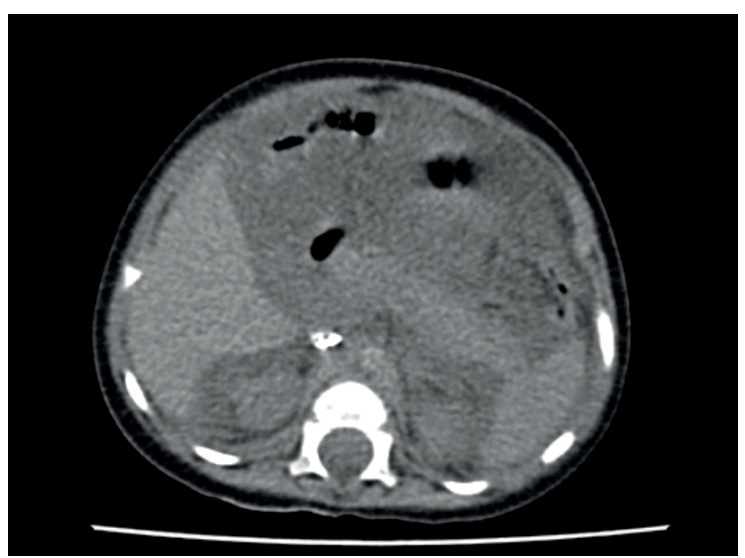

Fig. 1. Widespread free fluid in abdomen CT. (Case 1) on the appendix and the mesentery of small intestine. Histopathological sections of the biopsy materials showed focal necrosis, suppurative inflammation and fibrinous exudate in the omentum and the fat tissue, compatible with mesenteric panniculitis. Staining for tuberculosis and the culture was negative, and there was no IgG4-positive lymphoplasmocytic infiltration suggesting IgG4-related disease (Table I). An informed consent was received from the parents for publication.

\section{Case 2}

A ten-year-old boy with no previous disease was admitted to the emergency room due to abdominal pain and nausea for 2 weeks. On physical examination, he had tenderness with pain in the lower right quadrant. The examination of the other systems were normal. Laboratory tests revealed elevated leukocytes $\left(14100 / \mathrm{mm}^{3}\right)$, ESR $(27 \mathrm{~mm} / \mathrm{h})$, and CRP $(60.5$ $\mathrm{mg} / \mathrm{L})$. Urinalysis, liver and renal function tests were normal. X-ray was normal, but free fluid and an edematous appendix was detected in the abdominal ultrasonography. Perforated appendicitis was suspected; however, histopathological evaluation of the resected material was consistent with lymphoid hyperplasia and mesenteric panniculitis of the omentum. On further assessment, ANA was negative and C3, C4, IgG4 levels were normal. The patient was discharged and had no further complaints (Table I). An informed consent was received from the parents for publication.

\section{Case 3}

An eight-year-old girl was admitted to hospital because of vomiting and exhaustion. Past medical history revealed polyarticular JIA. She was on methotrexate and etanercept. She had inactive disease and had been off medications for 2 years. On admission, she had impaired renal functions and high acute phase response (CRP $18.8 \mathrm{mg} / \mathrm{L}$, ESR $103 \mathrm{~mm} / \mathrm{h}$ ). ANA was positive (1/1000-1/3200), extractable nuclear antibodies (ENA), ANCA, rheumatoid factor (RF), anti-cyclic citrullinated protein (antiCCP) were negative, C3 and C4 levels were normal. Bilateral hydronephrosis was detected on ultrasonography. The MRI revealed a mass 
Table I. Clinical Features and Treatment of 3 Cases with Mesenteric Panniculitis Diagnosis.

\begin{tabular}{llllll}
\hline Cases Age/Sex & Symptoms / Findings & Treatment & Result & Additional info \\
\hline 1 & $\begin{array}{l}5 \text { months/ } \\
\text { female }\end{array}$ & $\begin{array}{l}\text { Vomiting, abdominal } \\
\text { distention and } \\
\text { tenderness }\end{array}$ & Surgical & $\begin{array}{l}\text { Normal followup } \\
\text { without treatment }\end{array}$ & No fibrosis \\
2 & 10 years/male & $\begin{array}{l}\text { Abdominal pain, } \\
\text { nausea, vomiting }\end{array}$ & Surgical & $\begin{array}{l}\text { Normal followup No fibrosis } \\
\text { without treatment }\end{array}$ \\
3 & 15 years/male & $\begin{array}{l}\text { Vomiting, kidney } \\
\text { failure, hydronephrosis }\end{array}$ & $\begin{array}{l}\text { Surgical, } \\
\text { Steroid, Aza, } \\
\text { MTX, Anti-IL6 }\end{array}$ & $\begin{array}{l}\text { Remission with } \\
\text { treatment }\end{array}$ & $\begin{array}{l}\text { Fibrosis and } \\
\text { hydronephrosis } \\
\text { Polyarticular RF (-) JIA }\end{array}$ \\
\hline
\end{tabular}

Aza: Azathioprine, MTX: Methotrexate anti-IL 6: Tocilizumab, JIA: Juvenile idiopathic arthritis

surrounding perirenal tissue and ureters, leading to retroperitoneal fibrosis. (Fig. 2) Histopathologic examination resulted in fatty necrosis and septal fibrosis in the adipose tissue fragments, namely mesenteric panniculitis. (Fig. 3) Amyloid was negative.

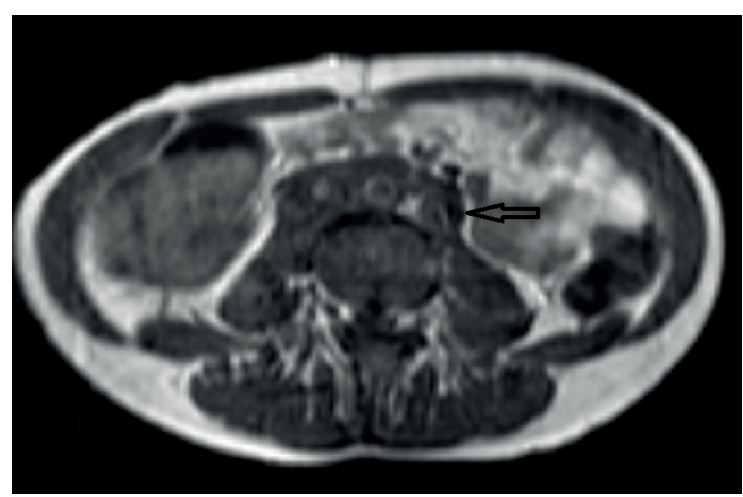

Fig. 2. The arrow shows the fibrosing mass surrounding the ureters. Abdominal MRI-T1. (Case 3)

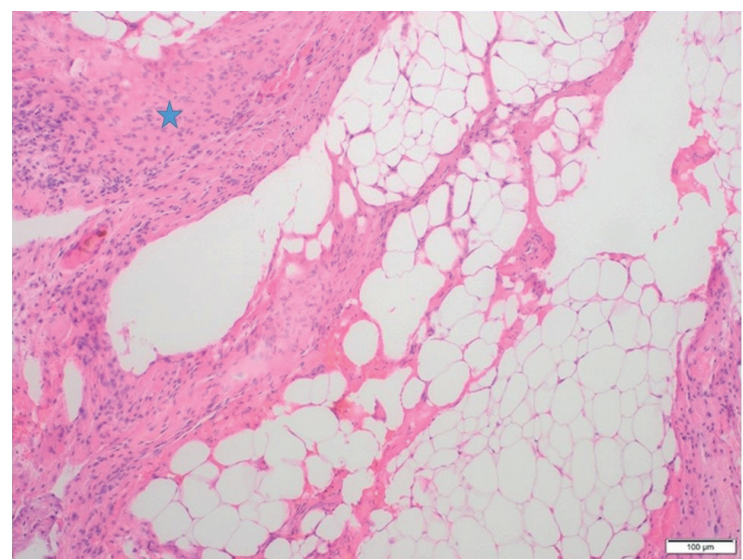

Fig. 3. Fat necrosis and septal fibrosis in adipose tissue fragments in sections. Chronic inflammation and fibrozis area (star). Hematoxylin-eosin stain x10 (Case 3)
Staining for IgG4 was negative. The serum IgG4 level was also normal. She had an initial response to steroid therapy, which was maintained by methotrexate and azathioprin. The disease was under control for 1.5 years, then steroids followed by tocilizumab was added to control the regrowing retroperitoneal fibrosis. The patient is still in remission for 2 years under tocilizumab treatment (Table I). An informed consent was received from the parents for publication.

\section{Discussion}

Mesenteric panniculitis, chronic fibrosing inflammation of the colon and intestinal mesentery was first reported as retractile sclerosing mesenteritis in 1924. Some experts believe that mesenteric panniculitis is an underdiagnosed condition, it is not mentioned in the differential diagnosis, and those patients are less reported. ${ }^{9}$ It is estimated that it affects $1 \%$ of the population. The frequency increases with age, especially in the 5 th and 7 th decade. ${ }^{10}$ It is rarely reported in children, probably due to lesser amount of mesenteric fat tissue compared to adults. ${ }^{11}$

Viswanathan et al. ${ }^{12}$ reported a 6-year-old girl with mesenteric panniculitis, and they reviewed 16 published pediatric cases. In this paper, the age range varied between 18 months and 12 years, with equal frequency of both sexes. A five-month old girl is the youngest case, according to the English literature. ${ }^{12}$ Hasosah et al. ${ }^{13}$ presented a case of a sevenyear-old girl who referred to the clinic with anorexia, weight loss, chronic diarrhea, and abdominal pain. Lymphoma, Crohn's disease and abdominal tuberculosis were considered 
as the preliminary diagnoses. A tissue biopsy taken with laparotomy operation reported mesenteric panniculitis associated with IgG4related disease. ${ }^{13}$ Two of our cases had no accompanying rheumatologic disease; however, they had acute abdomen which was diagnosed as mesenteric panniculitis. The third case who had a previous diagnosis of JIA was referred with hydronephrosis and renal failure, which was diagnosed as retroperitoneal fibrosis and mesenteric panniculitis.

Most histopathologic analyses demonstrate various degrees of fibrosis, chronic inflammation, fatty necrosis of the infiltrate without evidence of vascular involvement. Biopsy results of the pediatric series range between macroscopic increased fat tissue resulting in widespread panniculitis and fibrosis. ${ }^{12}$ The first case had adipose tissue necrosis, suppurative inflammation with fibrinous exudate. The second case had lymphoid hyperplasia and fibrolipomatous appearance. In the third case, there was adipose tissue fragments and septal fibrosis in addition to fatty necrosis. All of them had panniculitis with fibrosis.

Chen et al. ${ }^{14}$ mentioned that mesenteric panniculitis was seen as a possible subset of IgG4-associated sclerosing disorders because of increased IgG4 expression in related plasma cells. IgG4-related disease has not been detected in most cases in the literature, and our cases also do not have IgG4 association. ${ }^{15}$

The disease may be asymptomatic in $30-50 \%$ of cases or may have various findings. ${ }^{13,15}$ The most common symptoms in the reported articles are abdominal pain, bloating, and diarrhea, while nausea, vomiting, weight loss and constipation are seen less frequently. ${ }^{16-18}$ Rare presentations include rectal bleeding, jaundice, gastric outlet obstruction and acute abdomen. ${ }^{19}$ Mesenteric panniculitis is detected in patients with fever of unidentified origin (FUO). ${ }^{20}$ It is usually benign, but intestinal obstruction, perforation, pneumoperitoneum, ischemic colitis, chylous acid, vascular thrombosis, recurrent pleural effusion, biliary and pancreatic fistula are reported complications..$^{21}$ Because of this wide variety and non-specific clinical features, mesenteric panniculitis should be in the differential diagnosis of chronic abdominal pain in cases where common causes are excluded. Physical examination, laboratory and imaging investigation may not be sufficient alone for diagnosis. Similar to adults, the most common finding in children is abdominal pain, sometimes presenting as acute abdomen, leading to surgical intervention.,21-23 Two of the cases in this paper presented with signs and symptoms of acute abdomen, one of them had a preliminary diagnoses of appendicitis resulting in surgical intervention the other patient received an explorative laparotomy.

Laboratory findings are generally nonspecific and non-diagnostic. Most cases might have nonspecific elevations of inflammatory markers. ${ }^{15,24}$ Our cases also had elevated acute phase response due to inflammation.

Computed tomography (CT) scanning is the preferred imaging method for mesenteric panniculitis. $^{25}$ Daskalogiannaki et al. ${ }^{19}$ reviewed 7620 abdominal CT scans, and the prevalence of mesenteric panniculitis was found as $0.6 \%$. There are two characteristic features; "Sign of fat ring" and "tumoral pseudocapsule". ${ }^{21,25}$ However, CT findings are mostly non-specific and may require further evaluation with explorative laparotomy in pediatric patients. ${ }^{24}$ The exact diagnosis is confirmed by histopathological examination.

Most of the mesenteric panniculitis patients have spontaneous regression and no treatment is needed. ${ }^{9}$ Some symptomatic cases are responsive to the steroids, even resulting in complete remission. ${ }^{6}$ Azathioprine, cyclophosphamide, methotrexate, colchicine, tamoxifen, oral progesterone, thalidomide are the other treatments reported in the literature. Infliximab is used in a child with sclerosing mesenteritis with celiac disease. ${ }^{26}$ Biologics are rarely needed, particularly in patients with an underlying immune disease, as tocilizumab was effective in our patient with JIA. Surgical resection is performed for diagnostic and therapeutic purpose, in most pediatric cases. ${ }^{12}$

In conclusion, mesenteric panniculitis is a histopathological diagnosis with uncertain etiology. The disease has different faces with various clinical and radiological findings. In particular, surgeons and other clinicians should 
keep this disease in mind in the differential diagnosis of abdominal pain, with or without mass. The diagnosis is so difficult in some cases that explorative laparotomy is indicated. There is an increased awareness of the disease by using computerized tomography and/ or histopathological evaluation in children with chronic abdominal pain. Mesenteric panniculitis should be in the differential diagnosis of abdominal pain, especially if the patient has sign or symptoms of intestinal or urinary obstruction. Abdominal screening with CT or MRI and surgical consultation is necessary in these cases. The pathologist should be informed if histopathological examination is needed. There is no consensus on treatment, and the type of treatment should be tailored according to the patient. Once the diagnosis is established, a close follow up in the light of surgical consultation is appropriate. Spontaneous remission can be seen in some patients and surgery is an option in others. Immunosuppressive treatment may be required in cases with rheumatic disease and in recurrent cases. Larger series are needed to establish a detailed algorithm on treatment and disease follow-up.

\section{REFERENCES}

1. Nyberg L, Björk J Björkdahl P, Ekberg O, Sjöberk K, Vigren L. Sclerosing mesenteritis and mesenteric panniculitis-clinical experience and radiological features BMC Gastroenterol 2017; 17: 75.

2. Pinheiro F, Rgo A, ArajoFilho I. Mesenteric panniculitis in the elderly-update on diagnostic and therapeutic approch. Int J Surg Med 2016; 2: 127-133.

3. Hakgüder G, Akgür FM, Olguner M, Ozer E, Aktug T. A case of mesenteric panniculitis in a 4-year-old child. Pediatr Int 2000; 42: 577-578.

4. Schaffler A, Herfarth H. Creeping fat in Crohn's disease: travelling in a creeper lane of research? Gut 2005; 54: 742-744.

5. Dor AM, Kohler JL, Aubrespy P, Scheiner C, Pizzi M, Lebreuil G. Mesenteric panniculitis, an unusual initial stage of acute lupus erythematosus in a ten-year old girl. Arch Anat Cytol Pathol 1982; 30: 121-124.

6. Bae SH, Park SJ, Kim WS, Lee MW, Kim SJ. Mesenteric panniculitis in a thirtee-year-old Korean boy treated with prednisolone: A case report. Pediatr Gastroenterol Hepatol Nutr 2016; 19: 143-146.
7. Emory TS, Monihan JM, Carr NJ, Sobin LH. Sclerosing mesenteritis, mesenteric panniculitis and mesenteric lipodystrophy: a single entity? Am J Surg Pathol 1997; 21: 392-398.

8. Coulier B. Mesenteric panniculitis. Part 1: MDCTpictorial review. JBR-BTR 2011; 94: 229-240.

9. Nicholson JA, Smith D, Diab M, Scott MH. Mesenteric panniculitis in Merseyside: a case series and a review of the literature. Ann R Coll Surg Engl 2010; 92.

10. Ferrari TC, Couto CM, Vilaca TS, Xavier MA, Faria LC.An unusual presentation of mesenteric panniculitis. Clinics (Sao Paulo) 2008; 63: 843-844.

11. Issa I, Baydoun H. Mesenteric panniculitis: various presentations and treatment regimens. World J Gastroenterol 2009; 15: 3827-3830.

12. Viswanathan V, Murray KJ. Idiopathic sclerosing mesenteritis in paediatrics: Report of a successfully treated case and a review of literature. Pediatric Rheumatol Online J 2010; 8: 5.

13. Hasosah MY, Satti MB, Yousef YA, et al. IgG4-related sclerosing mesenteritis in a 7year-old Saudi girl. Saudi J Gastroenterol 2014; 20: 385-388.

14. Chen TS, Montgomery EA. Are tumefactive lesions classified as sclerosing mesenteritis a subset of IgG4related sclerosing disorders? J Clin Pathol 2008; 61: 1093-1097.

15. Delgado Plasencia L, Rodríguez Ballester L, LópezTomassetti Fernández EM, Hernández Morales A, Carrillo Pallarés A, Hernández Siverio N. Mesenteric panniculitis: experience in our center. Rev Esp Enferm Dig 2007; 99: 291-297.

16. Akram S, Pardi DS, Schaffner JA, Smyrk TC. Sclerosing mesenteritis: Clinical features treatment and outcome in ninety-two patients. ClinGastroenterol Hepatol 2007; 5: 589-596.

17. Kipfer RE, Moertel CG, Dahlin DC. Mesenteric lipodystrophy. Ann Intern Med1974; 80: 582-588.

18. Oztan OM, Ozdemir T, Uncel M, Diniz G, Koyluoğlu G. Isolated omental panniculitis in a child with abdominal pain. Case report. Arch Argent Pediatr 2016; 114: e425-e428.

19. Daskalogiannaki M, Voloudaki A, Prassopoulos P, et al. CT evaluation of mesenteric panniculitis: prevalence and associated diseases. AJR Am J Roentgenol 2000; 174: 427-431.

20. Hirano H, Yoshida A, Sasae Y, Sakuta T, Morita Y. Mesenteric panniculitis: a rare cause of fever. Int J Rheum Dis 2012; 15: e40-e42.

21. Rumman N, Rumman G, Sharabati B, Zagha R, Disi N. Mesenteric panniculitis in a child misdiagnosed as appendicular mass: a case report and review of literature. Springerplus 2014; 3: 73. 
22. Cakmak O, Tanyel FC, Cağlar M, Göğüs S. Mesenteric panniculitis mimicking acute abdomen in a 4-yearold child. Z Kinderchir 1986; 41: 313-314.

23. Duman M, Kocak O, Fazli O, Kocak C, Atici AE, Duman U. Mesenteric panniculitis patients requiring emergency surgery: Report of three cases. Turk J Gastroenterol 2012; 23: 181-184.

24. Liang CP, Yang M, Chen PY, Geng LL, Li DY, Gong ST. Scelorising mesenteritis in a 5-year-old Chinese boy: a case report. BMC Pediatr 2017; 17: 179.
25. Horton KM, Lawler LP, Fishman EK. CT findings in sclerosing mesenteritis (panniculitis): spectrum of disease. Radiographics 2003; 23: 1561-1567.

26. Sampert C, Lowichik A, Rollins M, Inman CJ, Bohnsack J, Pohl JF. Sclerosing mesenteritis in a child with celiac disease. J Pediatr Gastroenterol Nutr 2011; 53: 688-690. 